\title{
Reduced-dose whole-brain radiotherapy with tumor bed boost after upfront high-dose methotrexate for primary central nervous system lymphoma
}

\author{
Tae Hoon Lee ${ }^{1}$, Joo Ho Lee ${ }^{1}$, Ji Hyun Chang ${ }^{1}$, Sung-Joon Ye ${ }^{1}$, Tae Min Kim² ${ }^{2}$ Chul-Kee Park ${ }^{3}$, II Han Kim ${ }^{1,4}$, \\ Byoung Hyuck Kim ${ }^{5}$, Chan Woo Wee ${ }^{5}$ \\ ${ }^{1}$ Department of Radiation Oncology, Seoul National University Hospital, Seoul National University College of Medicine, Seoul, Korea \\ ${ }^{2}$ Department of Internal Medicine, Seoul National University Hospital, Seoul National University College of Medicine, Seoul, Korea \\ ${ }^{3}$ Department of Neurosurgery, Seoul National University Hospital, Seoul National University College of Medicine, Seoul, Korea \\ ${ }^{4}$ Cancer Research Institute, Seoul National University Hospital, Seoul National University College of Medicine, Seoul, Korea \\ ${ }^{5}$ Department of Radiation Oncology, Seoul Metropolitan Government Seoul National University Boramae Medical Center, Seoul, Korea
}

Received: January 29, 2020

Revised: March 17, 2020

Accepted: March 18, 2020

Correspondence:

II Han Kim

Department of Radiation Oncology, Seoul National University Hospital,

Seoul National University College of Medicine, 101, Daehak-ro, Jongno-

gu, Seoul 03080, Korea.

Tel: +82-2-2072-2528

Fax: +82-2-765-3317

E-mail: ihkim@snu.ac.kr

ORCID:

https://orcid.org/0000-0002-4755-5201
Purpose: This retrospective study compares higher-dose whole-brain radiotherapy (hdWBRT) with reduced-dose WBRT (rdWBRT) in terms of clinical efficacy and toxicity profile in patients treated for primary central nervous system lymphoma (PCNSL).

Materials and Methods: Radiotherapy followed by high-dose methotrexate (HD-MTX)-based chemotherapy was administered to immunocompetent patients with histologically confirmed PCNSL between 2000 and 2016. Response to chemotherapy was taken into account when prescribing the radiation dose to the whole brain and primary tumor bed. The whole brain dose was $\leq 23.4$ Gy for rdWBRT $(n=20)$ and $>23.4$ Gy for hdWBRT $(n=68)$. Patients manifesting cognitive disturbance, memory impairment and dysarthria were considered to have neurotoxicity. A median follow-up was 3.62 years. Results: The 3-year overall survival (OS) and progression-free survival (PFS) were 70.0\% and 48.9\% with rdWBRT, and $63.2 \%$ and $43.2 \%$ with hdWBRT. The 3-year OS and PFS among patients with partial response $(n=45)$ after chemotherapy were $77.8 \%$ and $53.3 \%$ with rdWBRT, and $58.3 \%$ and $45.8 \%$ with hdWBRT ( $p>0.05$ ). Among patients with complete response achieved during follow-up, the 3-year freedom from neurotoxicity (FFNT) rate was $94.1 \%$ with rdWBRT and $62.4 \%$ with hdWBRT. Among patients aged $\geq 60$ years, the 3 -year FFNT rate was $87.5 \%$ with rdWBRT and $39.1 \%$ with hdWBRT $(p=0.49$ ). Neurotoxicity was not observed after rdWBRT in patients aged below 60 years. Conclusion: rdWBRT with tumor bed boost combined with upfront HD-MTX is less neurotoxic and results in effective survival as higher-dose radiotherapy even in partial response after chemotherapy.

Keywords: Non-Hodgkin lymphoma, Central nervous system, Cranial irradiation, Adverse effects

\section{Introduction}

Primary central nervous system lymphoma (PCNSL) is a highly aggressive form of non-Hodgkin's lymphoma. Optimal treatment for PCNSL is disputed, but high-dose methotrexate (HD-MTX)-based chemotherapy followed by whole-brain radiotherapy (WBRT) remains a standard treatment strategy.
The major drawback of WBRT for PCNSL is the possibility of neurotoxicity $[1,2]$. Several approaches have been adopted to overcome or alleviate this limitation. One of the approaches entailed complete omission of WBRT. G-PCNSL-SG-1 is a phase III trial designed to test non-inferiority of HD-MTX-based chemotherapy alone, compared with chemotherapy followed by WBRT. Although there was no statistically significant difference in overall survival be-

Copyright@ 2020 The Korean Society for Radiation Oncology

This is an Open Access article distributed under the terms of the Creative Commons Attribution Non-Commercial License (http://creativecommons.org/licenses/by-nc/4.0/) which permits unrestricted non-commercial use, distribution, and reproduction in any medium, provided the original work is properly cited. 
tween treatment groups, the trial failed to reach predefined non-inferiority margin [3]. Another attempt to avoid WBRT involved substitution of WBRT for high-dose chemotherapy with autologous stem cell transplantation. Recent trials show that this approach reduces neurotoxicity due to WBRT although the risk of hematologic toxicity and treatment-related death is increased $[4,5]$.

While these trials omitted WBRT, several attempts were also made to reduce neurotoxicity despite WBRT treatment efficacy as consolidation treatment, by reducing the radiation dose. Morris et al. [6] conducted a multi-center phase II study to reduce the dosage of WBRT in patients who achieved complete response after chemotherapy, with adequate overall survival (OS) and minimal neurotoxicity. Our group showed promising results of dose reduction in WBRT with tumor bed boost, with $59.8 \%$ of 3-year OS rate and $47.9 \%$ of 3 -year progression-free survival (PFS) rate [2]. Furthermore, clinicians have implemented dose reduction of WBRT in patients with a favorable response to chemotherapy, as shown in KROG 14-20 study in which no more than 5\% of patients underwent WBRT with a dose less than $30 \mathrm{~Gy}$ in early 2000s, whereas more than 25\% of patients underwent WBRT with such dose in 2010 [7]. Although this dose reduction is prevalent, the comparison of treatment outcomes or toxicity profile based on differences in WBRT dosage has yet to be reported. The purpose of the present study is to show that dose reduction of WBRT after upfront HDMTX is safe. Towards this end, the study compared clinical efficacy and toxicity profile of low-dose WBRT and tumor bed boost with that of higher-dose WBRT and tumor bed boost in PCNSL patients who underwent upfront HD-MTX-based chemotherapy.

\section{Materials and Methods}

\section{Study population}

This study was approved by the Institutional Review Board of Seoul National University Hospital before collecting patient information (No. 1904-072-1026). A retrospective review was undertaken using medical records of pathologically confirmed PCNSL patients who underwent upfront HD-MTX-based chemotherapy followed by WBRT from January 2000 to December 2016. Patients with immunocompromised state, previous cancer history, or disease involving extra-central nervous system (CNS) at the time of presentation were excluded. WBRT with dose lower than or equal to 23.4 Gy was defined as reduced-dose WBRT (rdWBRT), and therapy with dose higher than 23.4 Gy was defined as higher-dose WBRT (hdWBRT). The rdWBRT was administered to 20 out of 88 eligible patients. Characteristics of the patients and treatments administered to both groups are summarized in Table 1.
Table 1. Patients' characteristics

\begin{tabular}{|c|c|c|c|}
\hline Characteristic & $\begin{array}{c}\text { Reduced-dose } \\
\text { WBRT }\end{array}$ & $\begin{array}{c}\text { Standard-dose } \\
\text { WBRT }\end{array}$ & $p$-value \\
\hline Age at diagnosis (yr) & $\begin{array}{c}58.7 \\
(46.2-75.2)\end{array}$ & $\begin{array}{c}56.1 \\
(23.4-75.1)\end{array}$ & 0.599 \\
\hline$<60$ & $11(55.0)$ & $44(64.7)$ & \\
\hline$\geq 60$ & $9(45.0)$ & $24(35.3)$ & \\
\hline Sex & & & 0.852 \\
\hline Male & $12(60.0)$ & $37(54.4)$ & \\
\hline Female & $8(40.0)$ & $31(45.6)$ & \\
\hline ECOG performance status & & & 0.683 \\
\hline $0-1$ & $14(70.0)$ & $42(61.8)$ & \\
\hline $2-4$ & $6(30.0)$ & $26(38.2)$ & \\
\hline Pathology & & & 0.399 \\
\hline $\begin{array}{l}\text { Diffuse large B-cell lym- } \\
\text { phoma }\end{array}$ & $17(85.0)$ & 64 (94.1) & \\
\hline Other B-cell lymphoma & $1(5.0)$ & $1(1.5)$ & \\
\hline T-cell lymphoma & $2(10.0)$ & $3(4.4)$ & \\
\hline Eye involvement & & & 0.134 \\
\hline Yes & $3(15.0)$ & $2(2.9)$ & \\
\hline No & $17(85.0)$ & $66(97.1)$ & \\
\hline CSF involvement & & & 0.703 \\
\hline Yes & $3(15.0)$ & $6(8.8)$ & \\
\hline No & $17(85.0)$ & $62(91.2)$ & \\
\hline Operation & & & 0.190 \\
\hline Stereotactic biopsy & $20(100)$ & $58(85.3)$ & \\
\hline Gross total resection & $0(0.0)$ & $7(10.3)$ & \\
\hline Subtotal resection & $0(0.0)$ & $3(4.4)$ & \\
\hline Number of lesions & & & 1.000 \\
\hline Single & $5(25.0)$ & $19(27.9)$ & \\
\hline Multiple & $15(75.0)$ & $49(72.1)$ & \\
\hline Deep structure involvement ${ }^{a)}$ & & & 0.864 \\
\hline Yes & $14(70.0)$ & $44(64.7)$ & \\
\hline No & $6(30.0)$ & $24(35.3)$ & \\
\hline LDH elevation & & & $0.640^{\mathrm{b})}$ \\
\hline Yes & $6(30.0)$ & $27(39.7)$ & \\
\hline No & $13(65.0)$ & $39(57.4)$ & \\
\hline Not reported & $1(5.0)$ & $2(2.9)$ & \\
\hline Rituximab usage & & & 0.003 \\
\hline Yes & $10(50.0)$ & $10(14.7)$ & \\
\hline No & $10(50.0)$ & $58(85.3)$ & \\
\hline Post-radiation cytarabine & & & 0.003 \\
\hline Yes & $12(60.0)$ & $15(22.1)$ & \\
\hline No & $8(40.0)$ & $53(77.9)$ & \\
\hline
\end{tabular}

Values are presented as median (range) or number (\%). WBRT, whole-brain radiotherapy; ECOG, Eastern Cooperative Oncology Group; CFS, cerebrospinal fluid; LDH, lactate dehydrogenase.

${ }^{a)}$ Deep structure was defined as basal ganglia, corpus callosum, brain-

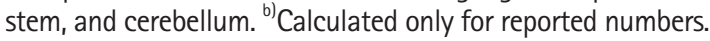




\section{Chemotherapy}

As specified in eligibility criteria, all patients were treated with HD-MTX-based chemotherapy with median 5 cycles (range, 2 to 6 cycles). Exact chemotherapy regimen was varied depending on the medical oncologist decision and treatment period. Eighty-one patients (92.0\%) underwent combination chemotherapy with methotrexate, vincristine, and procarbazine, which was similar to that used in the RTOG 93-10 study [8]. Until 2004, a regimen without procarbazine was also used in $6(5.8 \%)$ patients. One patient (1.1\%) with T-cell lymphoma was treated with HD-MTX and cytarabine. The optimal dose of methotrexate was $2.5 \mathrm{~g} / \mathrm{m}^{2}$ at least, but a lower dose was used in 5 patients (5.7\%) with a history of liver cirrhosis or chronic kidney disease. The range of methotrexate dose was $1.5-4.0 \mathrm{~g} / \mathrm{m}^{2}$, and a median of 5 cycles was administered (range, 2 to 6 cycles). Methotrexate was infused on the first day of each cycle, every 2 to 3 weeks. Vincristine $1.4 \mathrm{mg} / \mathrm{m}^{2}$ was administered on the first day of each cycle, and procarbazine 100 $\mathrm{mg} / \mathrm{m}^{2}$ was administered orally for 7 days on $1 \mathrm{st}, 3 \mathrm{rd}$, and 5 th cycles. Since 2014 , the additional use of rituximab became routine. Twenty patients were treated with rituximab (R-MVP [rituximab, methotrexate, procarbazine, and vincristine] regimen) and most of them started treatment from 2014. After completion of radiotherapy, consolidation cytarabine was added to 27 patients (30.7\%), and most of them were treated from 2011. Because additional patients underwent rdWBRT subsequently, a higher ratio of patients was treated with rituximab or post-radiation cytarabine, as shown in Table 1. Three patients (3.4\%) underwent concurrent chemotherapy with temozolomide, and 16 patients (18.2\%) with positive cerebrospinal fluid (CSF) cytology or suspicious leptomeningeal involvement were treated with intrathecal methotrexate.

\section{Radiotherapy}

Radiotherapy started 3 to 6 weeks after HD-MTX chemotherapy. Doses to the whole brain and tumor bed were mainly based on radiological response to upfront chemotherapy. As shown in Table 2, the whole brain dose or total dose was lower $(p<0.05)$ in the rdWBRT group for each category of responses (complete response $[C R]$, partial response [PR], or stable disease/progressive disease [SD/ PD]) to upfront chemotherapy. The proportion of patients who received rdWBRT was definitely higher than those treated prior to 2011 (Fig. 1); only 3 (5.8\%) among 52 patients were treated before 2011 while 17 (47\%) among 36 patients were treated subsequently.

A three-dimensional conformal therapy (3D-CRT) based on conventional fractionation of daily 1.8 Gy of 4-6 MV X-rays was administered to 65 patients (73.9\%), intensity-modulated radiotherapy (IMRT) was provided to 14 patients (15.9\%) since late 2014. One patient underwent WBRT as 3D-CRT then tumor bed boost as
Table 2. Radiation dosage in different treatment groups based on response to chemotherapy

\begin{tabular}{lll}
\hline Response after chemotherapy & WBRT dose (Gy) & Total dose (Gy) \\
\hline Standard-dose group (> 23.4 Gy) & & \\
CR $(n=27)$ & $27.0(25.2-30.6)$ & $45.0(36.0-54.0)$ \\
PR $(n=36)$ & $30.6(25.2-36.0)$ & $50.4(45.0-55.8)$ \\
SD/PD $(n=5)$ & $30.6(27.0-36.0)$ & $50.4(45.0-54.0)$ \\
Reduced-dose group ( $\mathrm{n} 23.4$ Gy) & & \\
CR $(n=9)$ & $19.8(18.0-23.4)$ & $36.0(36.0-50.4)$ \\
PR $(n=9)$ & $21.6(18.0-23.4)$ & $45.0(45.0-50.4)$ \\
SD/PD $(n=2)$ & $20.7(18.0-23.4)$ & $42.3(39.6-45.0)$ \\
\hline
\end{tabular}

Values are presented as median (range).

WBRT, whole-brain radiotherapy; $C R$, complete response; $P R$, partial response; $\mathrm{SD}$, stable disease; $\mathrm{PD}$, progressive disease.

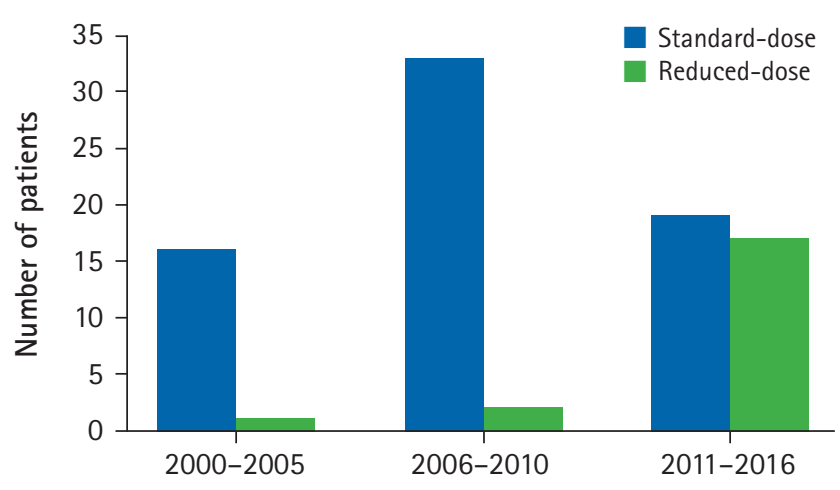

Fig. 1. Histogram representing treatment groups over different time periods.

IMRT, while 10 patients underwent WBRT as IMRT then tumor bed boost as 3D-CRT. For 3 patients, IMRT was applied to both WBRT and tumor bed boost. Boost to tumor bed was given sequentially, after WBRT was done. The gross tumor volume was delineated based on pre-chemotherapy tumor volume. The clinical target volume margin for tumor bed boost was 0.5 to $1.5 \mathrm{~cm}$, and the planning target volume margin was mostly $0.3 \mathrm{~cm}$. Whole-spine radiotherapy was administered to 5 patients (5.7\%) with positive CSF cytology. Range of dose to whole-spine was 19.8 to $36 \mathrm{~Gy}$.

\section{Follow-up and evaluation of neurotoxicity}

The median follow-up duration was 3.62 years (range, 0.38 to 19.2 years) in all patients; 3.62 years (range, 1.12 to 7.27 years) for rdWBRT, and 3.60 years (range, 0.38 to 19.2 years) for hdWBRT. Follow-up visit and response evaluation was done every 3 to 4 months for 2 years, every 6 months until 5 years from the end of the therapy, and yearly thereafter. Survival data was retrieved from the national registration system of Korean government.

Radiological response was evaluated after chemotherapy, one 
month after radiotherapy, and at every subsequent follow-up. Contrast-enhanced magnetic resonance with or without fluid-attenuated inversion recovery (FLAIR), diffusion-, perfusion- or susceptibility-weighted image (SWI) was administered. Response was evaluated according to international response criteria for PCNSL [9].

Neurotoxicity was evaluated via retrospective analysis of medical records. To exclude disease-related effects, patients who achieved CR within the follow-up period were included in this toxicity analyses. Treatment-related neurotoxicity was defined as occurrence of grade 2 or higher cognitive disruption, memory impairment or dysarthria after completion of radiotherapy. The neurotoxicity was graded by the Common Terminology Criteria for Adverse Events (CTCAE) ver. 5.0.

\section{Endpoints and statistical analysis}

The study endpoints were OS, PFS, and freedom from neurotoxicity (FFNT), which were calculated by Kaplan-Meier method. OS and DFS were measured from the date of biopsy or resection for histological diagnosis, for each defined event. The OS event was defined as death of the patient, and the PFS event was defined as disease progression or death. To measure FFNT, time to occurrence of neurotoxicity was calculated from the completion of radiotherapy and censored at the date of last follow-up or the event. The log-rank test was conducted to compare treatment outcomes and toxicity profiles of different treatment groups. Chi-square test were performed to determine differences between categorical values, while Student t-test was used for continuous values. Multivariate Cox proportional hazards model was constructed by including variables considered as potential independent predictors ( $p$ $<0.05$ ) based on univariate analysis. Treatment group according to WBRT dose was always included in multivariate analysis, since this factor was mainly evaluated in this study. Univariate and multivariate Cox analyses included variables, such as prognostic and treatment-related factors, which showed differences between treatment groups. All of the statistical analyses were performed using R 3.6.0 (https://www.R-project.org/).

\section{Results}

\section{Response}

CR after HD-MTX based chemotherapy was observed in 36 (40.9\%) of all patients ( $\mathrm{n}=88)$, in $61(69.3 \%)$ patients 1 month after completion of radiotherapy, and in 75 (85.2\%) patients during the overall follow-up eventually.

\section{Survival analysis}

The 3- and 5-year OS rates were $64.8 \%$ and $52.9 \%$ for all the pa- tients, respectively; $70.0 \%$ and $64.2 \%$ for rdWBRT, respectively, compared with $63.2 \%$ and $50.6 \%$ for hdWBRT, respectively. The OS differences between the two treatment groups were not statistically significant ( $p=0.77$ ). Univariate Cox analysis revealed age ( $<60$ vs. $\geq 60$ years), involvement of deep structures, dose of methotrexate, number of HD-MTX cycles, administration of postoperative cytarabine, and $\mathrm{CR}$ after radiotherapy as potential independent predictors. In multivariate Cox analysis, age ( $p=0.0014)$, involvement of deep structure ( $p=0.017)$, and CR within follow-up period ( $p=0.00048$ ) were associated with OS, whereas treatment group by WBRT dose was not.

The 3- and 5-year PFS rates were $46.9 \%$ and $32.6 \%$ for all the patients, respectively; $48.9 \%$ and $15.3 \%$ with rdWBRT, respectively, and $46.4 \%$ and $35.2 \%$ with hdWBRT, respectively. No significant differences in PFS were found between the two groups ( $p=$ 0.80). The 5-year PFS for rdWBRT group might appear to be low; however, the 5-year number at risk was 1 , so this rate is not representative. Age, involvement of deep structures, and CR within the follow-up period were potential independent predictors in univariate Cox analysis. Age ( $p=0.013)$ and CR within the follow-up period ( $p=0.00022$ ) were associated with PFS in multivariate analysis. Treatment group based on WBRT dose was not associated with PFS. The OS and PFS based on WBRT doses in all the patients are presented in Fig. 2.

The patterns of failure did not differ by treatment groups. In the hdWBRT group, 35 (51.5\%) showed in-brain recurrence, which included 23 (33.8\%) cases of recurrence in irradiated tumor bed. CSF failure was detected in $10(14.7 \%)$ cases, and $13(19.1 \%)$ showed distant recurrence. In the rdWBRT group, 11 (55.0\%) had in-brain recurrence, and 5 (25.0\%) failed in irradiated tumor bed; 3 cases (15.0\%) showed CSF failure, and 4 (20.0\%) had distant recurrence. There was no significant difference between two groups $(p>0.05)$

The effect of WBRT dose on survival of PR patients after chemotherapy was analyzed. Among 45 patients (51.1\%) with PR, 9 underwent rdWBRT while 36 underwent hdWBRT. In the rdWBRT group, 3- and 5-year OS rates were $77.8 \%$ and $77.8 \%$, respectively, and in the hdWBRT group, the rates were 58.3\% and $40.0 \%$, respectively. The OS difference was not significant ( $p=0.33$ ). In terms of PFS, 3- and 5-year rates were 53.3\% and 20.0\%, respectively, in the rdWBRT group, and were $45.8 \%$ and $29.6 \%$ with hdWBRT. These differences in PFS between the two groups were not significant $(p=0.94)$. The survival patterns of PR patients based on WBRT dose are shown in Fig. 3.

Treatment outcomes according to post-radiation cytarabine were also evaluated. For 27 patients with post-radiation cytarabine, the 3- and 5-year OS rates were $77.8 \%$ and 56.0\%, respec- 

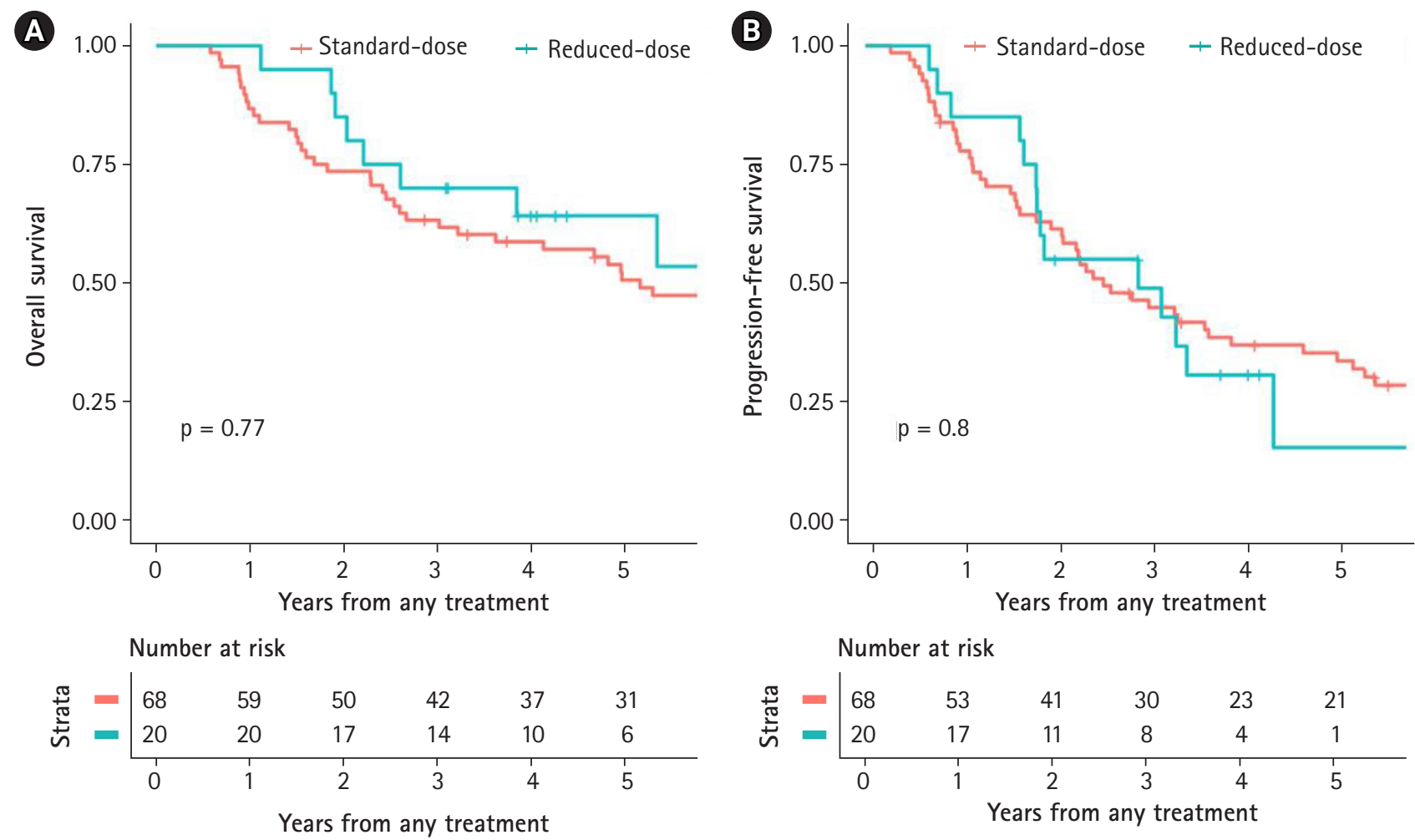

Fig. 2. Overall survival (A) and progression-free survival (B) of patients, based on dosage of whole-brain radiotherapy.
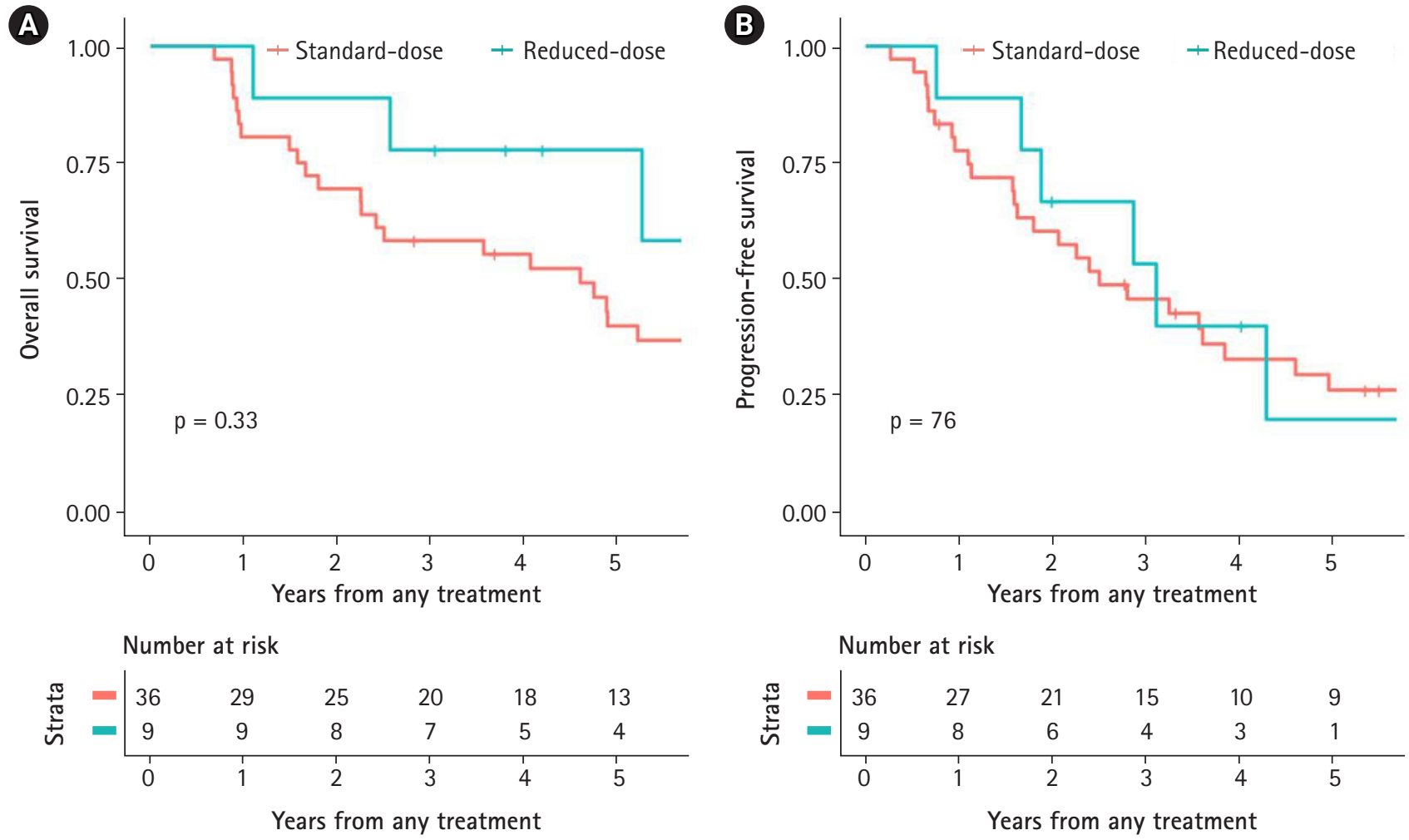

Fig. 3. Overall survival (A) and progression-free survival (B) of patients who achieved partial response, by dose of whole-brain radiotherapy. 
tively; and the 3- and 5-year PFS rates were 59.0\% and 30.7\%, respectively. In the remaining 61 patients, the 3- and 5-year OS rates were 59.0\% and 50.4\%, respectively; and the 3-and 5-year PFS rates were $41.8 \%$ and $32.7 \%$, respectively. A marginally significant difference was observed in OS ( $p=0.083)$ but not in PFS ( $p=0.38$ ) between the two groups.

\section{Neurotoxicity}

As previously stated, a total of 75 patients eventually achieved CR within the follow-up period, and these patients were included in neurotoxicity analyses. The 3-year FFNT rate was 69.7\%. At the time of diagnosis, 45 patients were younger than age 60 years whereas 30 patients were not. The 3-year FFNT rate was $77.3 \%$ in the young ( $<60$ years) and $55.6 \%$ in the older patients ( $p=$ 0.081). Fig. 4 shows Kaplan-Meier curves of FFNT rates by the age of 60 years.

Among patients included in neurotoxicity analyses, 18 patients were included in the rdWBRT group, and 57 were in the hdWBRT group. The 3-year FFNT rate was 94.1\% for rdWBRT group and $62.4 \%$ for hdWBRT group ( $p=0.33$ ). Univariate Cox analysis revealed that age, sex, multifocal involvement, involvement of deep structures, and methotrexate dose were potential independent predictors, but only age (hazard ratio $=1.05 ; 95 \%$ confidence interval, $1.00-1.11 ; p=0.038$ ) showed significant association in
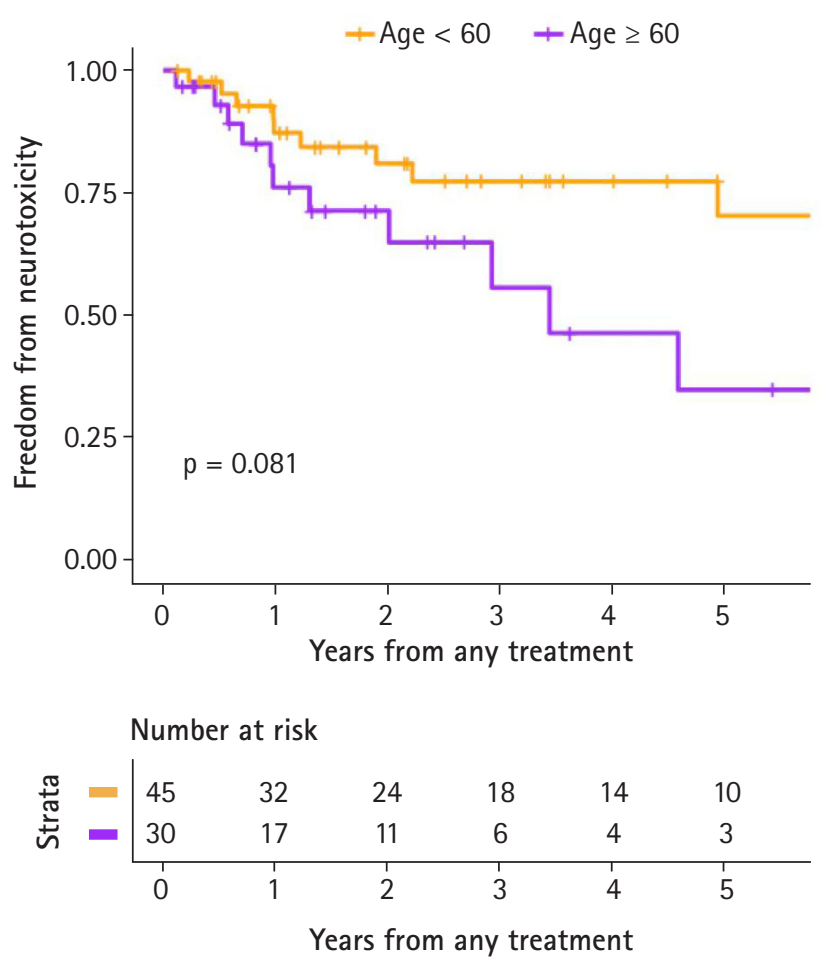

Fig. 4. Freedom from neurotoxicity rates, by age. multivariate analysis.

Among the young ( $<60$ years), the 3-year FFNT rate was $100 \%$ for rdWBRT group and $72.1 \%$ in the hdWBRT group ( $p=0.14$ ). No neurotoxicity was reported within the follow-up period in younger patients who underwent rdWBRT. Among the old ( $\geq 60$ years), the 3 -year FFNT rate was $87.5 \%$ in the rdWBRT group and $39.1 \%$ in the hdWBRT group ( $p=0.49$ ). Fig. 5 shows Kaplan-Meier curves of FFNT rates by WBRT dose.

\section{Discussion and Conclusion}

The present retrospective study revealed that reduced radiation dose administered to the whole brain and the primary tumor bed after upfront MTX-based chemotherapy resulted in OS and PFS rates similar to hdWBRT, and less neurotoxicity especially in patients younger than age 60 years. As there are no differences in treatment outcomes between rdWBRT and hdWBRT, radiotherapy with dose reduction is safe and feasible.

Our previous report demonstrated that HD-MTX-based chemotherapy followed by low-dose WBRT with tumor bed boost was an effective strategy for treatment of PCNSL [2]. Other groups also evaluated the efficacy of this strategy in patients who achieved CR after chemotherapy. Shah et al. [10] conducted a prospective trial to examine the effectiveness of dose reduction in WBRT with HD-MTX-based chemotherapy. Among 30 patients enrolled in the trial, 21 achieved CR after chemotherapy and 19 received WBRT with a dose of $23.4 \mathrm{~Gy}$. This group reported 2-year OS and PFS rates of $67 \%$ and $57 \%$, respectively. In patients undergoing rdWBRT, the 2-year OS and PFS rates were $89 \%$ and $79 \%$, respectively. Prospective evaluation of neurotoxicity revealed no treatment-related toxicity. The same group, Morris et al. [6], published their results of next phase of this trial. A total of 52 patients were recruited, and 31 patients achieved CR after initial chemotherapy. These patients underwent rdWBRT with a dose of $23.4 \mathrm{~Gy}$. The 2 -year PFS in this group was 77\%, and the 5-year OS was $80 \%$. Prospective cognitive evaluation revealed no functional decline after radiotherapy except in motor speed. Based on these results, the randomized RTOG 1114 study is evaluating the efficacy of rdWBRT by comparing chemoradiation with chemotherapy alone.

There was another attempt to reduce the dose of WBRT ended in failure. Bessell et al. [11] reported the results of prospective trial to reduce the dose of WBRT to 30.6 Gy in patients who achieved $\mathrm{CR}$ after chemotherapy. Chemotherapy regimens used in the trial consisted of cyclophosphamide, doxorubicin, vincristine and dexamethasone (CHOD)/carmustine, vincristine, methotrexate and cytarabine (BVAM). These regimens were not HD-MTX based, as $\mathrm{CHOD}$ regimen did not include methotrexate, and BVAM regimen 

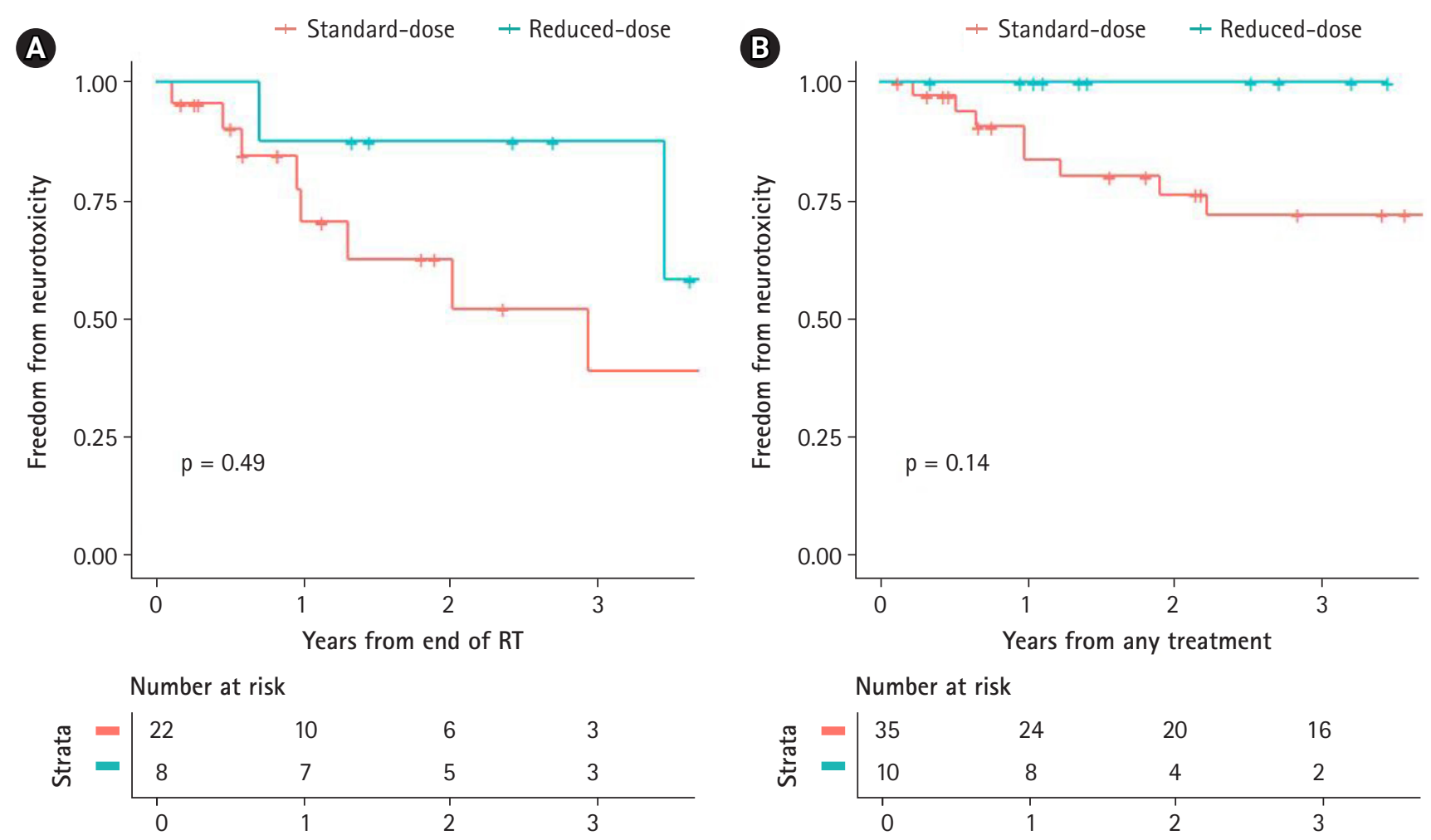

Fig. 5. Freedom from neurotoxicity by dose of whole-brain radiotherapy, patients with age older than or equal to 60 years (A) and younger than 60 years $(B)$.

included a methotrexate dose of $1.5 \mathrm{~g} / \mathrm{m} 2$, which was substantially lower than the HD-MTX regimen currently used [12]. Among patients who were younger than 60 years and achieved $C R$ after all the therapy, the 3-year OS was $92 \%$ in those exposed to $45 \mathrm{~Gy}$ of WBRT, while the rate was $60 \%$ in those treated with 30.6 Gy of WBRT, and this difference were statistically significant ( $p=0.04$ ). Because of the differences in chemotherapy regimen, the result should be interpreted with caution.

Nevertheless, there is already a tendency toward dose reduction in WBRT. As stated before, Koh et al. [7] conducted a multi-institutional retrospective study using decreased WBRT dose over time. In 2000, most patients included in the study underwent WBRT with a dose exceeding $40 \mathrm{~Gy}$; however, in 2010 nearly 25\% of patients underwent WBRT with a dose lower than $30 \mathrm{~Gy}$. This trend was also observed the present study, including a higher number of recent patients in rdWBRT group than the other groups.

The present study showed the possibility of rdWBRT with tumor bed boost in patients who achieved PR after chemotherapy without increasing the risk of relapse, as there was no difference in treatment outcomes based on WBRT dose. Similar to this study, other studies investigating WBRT dose reduction in patients who achieved non-CR after chemotherapy. Park et al. [13] retrospec- tively reviewed data of 62 patients aged younger than 60 years, and histologically confirmed diffuse B-cell lymphoma in CNS. Among these patients, 20 achieved partial response after chemotherapy and 17 underwent WBRT. Five patients were treated with 36 Gy of WBRT, while 12 were treated with 45 Gy. Three patients received a focal boost. The 3-year OS of patients in the $36 \mathrm{~Gy}$ and 45 Gy groups was 100\% and 83.3\%, respectively, with no significant difference in treatment outcomes based on WBRT dose. Sheu et al. [14] retrospectively investigated the records of 22 patients who underwent WBRT after HD-MTX-based chemotherapy for PCNSL. Patients who achieved CR or PR after chemotherapy were treated with a median dose 23.4 Gy of WBRT, while patients who had SD or PD, the median dose was 30.6 Gy. Eight non-CR patients received sequential radiation boost. There was no difference between CR and PR groups in terms of OS and freedom from CNS relapse rate. Together with the present study, these results show the possibility of wider usage of rdWBRT in PCNSL treatment.

Nevertheless, several aspects of dose reduction strategy involving WBRT remain to be addressed in the absence of consensus regarding the exact limit of low-dose WBRT associated with safety and efficacy [15]. Further, only a small number of patients underwent prospective neurocognitive tests in previous trials. For in- 
stance, in phase II trial of Morris et al. [6], only 12 patients were included in neurocognitive function analysis. Concerns about delayed neurotoxicity in rdWBRT remain to be addressed [16]. These issues will be addressed in further follow-up and ongoing trials such as RTOG 1114.

Neuronal damage of hippocampus due to radiation is known to be related to CNS toxicity [17]. Initially, hippocampus-sparing WBRT (hsWBRT) was applied for brain metastases, and clinical data showed that avoidance of hippocampus preserved memory function [18]. Using volumetric arc therapy (VMAT), one of the variations of IMRT, hsWBRT can be delivered safely and effectively [19]. In this study, 9 patients underwent unilateral or bilateral hsWBRT, in the absence of disease involvement near hippocampus, which resulted in comparable treatment outcomes. The 3-year OS and PFS rates of these patients were $77.8 \%$ and $55.6 \%$, respectively, and 3-year FFNT rate was 87.5\%. The feasibility of hsWBRT for PCNSL cannot be determined based on this study alone. Additional studies with a larger number of patients and longer follow-up periods may show lower rates of neurotoxicity rate as well as demonstrate the effectiveness of this approach.

As previously stated, elderly patients ( $\geq 60$ years) are vulnerable to neurotoxicity induced by WBRT. A few clinicians insist that although G-PCNSL-SG-1 trial failed to demonstrate the non-inferiority of completely omitting radiotherapy, it also showed a low clinical benefit of radiotherapy [3,20], as there was no significant difference in OS. Therefore, due to the risk of neurotoxicity in elderly patients, clinicians often omit radiotherapy in these settings [21]. The detrimental effect of WBRT is clear; however, currently there is no clear evidence supporting the omission of radiotherapy without compromising clinical outcomes. Also, a recent meta-analysis showed that WBRT improved clinical outcomes in elderly patients unlike more aggressive chemotherapy [22]. Another consolidation strategy without compromising cognitive function involves autologous stem cell transplantation [4,5]; however, elderly patients are not always amenable to this approach due to comorbidities and hematologic toxicity. Instead, rdWBRT may be indicated, as it ensures clinical outcomes with less neurotoxicity. Further reduction of WBRT dose or involved-field radiotherapy alone might be feasible for elderly patients. Shibamoto et al. [23] showed that partial brain irradiation with generous margins might be effective, and advances in imaging and radiation techniques may facilitate this strategy. These approaches are currently under investigation.

Although the rates were lower than in elderly patients, reports suggest modest incidence of delayed neurotoxicity in young $(<60$ years) patients exposed to WBRT [24]. Autologous stem cell transplantation was implemented due to the risk of long-term neuro- toxicity associated with WBRT. Due to the high rates of toxicity associated with intense chemotherapy, fit and young patients were subjected to this approach. For instance, the inclusion criteria of PRECIS trial stated that patients with age 18 to 60 were eligible [5]. While this trial showed excellent treatment outcomes and neurotoxicity profile in selected patients, 5 patients died after intense chemotherapy due to toxicity. Even though further evaluation is needed, this study reported no neurotoxicity in young patients within the follow-up period. Based on a risk-benefit analysis, rdWBRT might be appropriate for patients aged younger than 60 years.

One of the major limitations of this study is the retrospective nature of neurocognitive function assessment, as this study relies solely on review of medical records. Unreported or undetected treatment-related neurotoxicity might lead to overestimation of FFNT rate in this study. Also, due to the constant progression in treatment strategy of PCNSL, applied treatments were changed during the study period. In addition, concerns of potential bias existed due to unbalanced treatment between different groups based on WBRT dose. Despite these limitations, this study is still informative considering the relative rarity of the disease, the decent number of patients evaluated, and relatively consistent radiotherapy over the study period.

In conclusion, rdWBRT with tumor bed boost combined with upfront HD-MTX is less neurotoxic and results in effective survival as higher-dose radiotherapy even in PR after chemotherapy. Treatment with rdWBRT ( $\leq 23.4$ Gy) combined with HD-MTX showed no statistically significant difference compared with hdWBRT in terms of OS and PFS. Also, patients aged younger than 60 years and exposed to rdWBRT did not show neurotoxicity in present study, which indicates that these group of patients might benefit from rdWBRT.

\section{Conflict of Interest}

No potential conflict of interest relevant to this article was reported.

\section{References}

1. Gavrilovic IT, Hormigo A, Yahalom J, DeAngelis LM, Abrey LE. Long-term follow-up of high-dose methotrexate-based therapy with and without whole brain irradiation for newly diagnosed primary CNS Iymphoma. J Clin Oncol 2006;24:4570-4.

2. Kim BH, Kim IH, Park SH, et al. Low-dose whole brain radiotherapy with tumor bed boost after methotrexate-based chemotherapy for primary central nervous system Iymphoma. Cancer Res Treat 2014:46:261-9. 


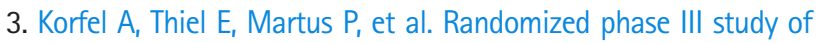
whole-brain radiotherapy for primary CNS Iymphoma. Neurology 2015;84:1242-8.

4. Ferreri AJM, Cwynarski K, Pulczynski E, et al. Whole-brain radiotherapy or autologous stem-cell transplantation as consolidation strategies after high-dose methotrexate-based chemoimmunotherapy in patients with primary CNS lymphoma: results of the second randomisation of the International Extranodal Lymphoma Study Group-32 phase 2 trial. Lancet Haematol 2017;4: e510-e523.

5. Houillier C, Taillandier L, Dureau S, et al. Radiotherapy or Autologous stem-cell transplantation for primary CNS Iymphoma in patients 60 years of age and younger: results of the Intergroup ANOCEF-GOELAMS Randomized Phase II PRECIS Study. J Clin Oncol 2019;37:823-33.

6. Morris PG, Correa DD, Yahalom J, et al. Rituximab, methotrexate, procarbazine, and vincristine followed by consolidation reduced-dose whole-brain radiotherapy and cytarabine in newly diagnosed primary CNS lymphoma: final results and long-term outcome. J Clin Oncol 2013;31:3971-9.

7. Koh HK, Kim IH, Kim TM, et al. Role of radiation therapy in primary central nervous system lymphoma : KROG 14-20 Collaborative Study of Brain and Lymphoma Committee. J Neurooncol 2017;135:629-38.

8. DeAngelis LM, Seiferheld W, Schold SC, Fisher B, Schultz CJ; Radiation Therapy Oncology Group Study 93-10. Combination chemotherapy and radiotherapy for primary central nervous system Iymphoma: Radiation Therapy Oncology Group Study 93-10. J Clin Oncol 2002;20:4643-8.

9. Abrey LE, Batchelor TT, Ferreri AJ, et al. Report of an international workshop to standardize baseline evaluation and response criteria for primary CNS Iymphoma. J Clin Oncol 2005;23:5034-43.

10. Shah GD, Yahalom J, Correa DD, et al. Combined immunochemotherapy with reduced whole-brain radiotherapy for newly diagnosed primary CNS lymphoma. J Clin Oncol 2007;25:4730-5.

11. Bessell EM, Lopez-Guillermo A, Villa S, et al. Importance of radiotherapy in the outcome of patients with primary CNS lymphoma: an analysis of the CHOD/BVAM regimen followed by two different radiotherapy treatments. J Clin Oncol 2002;20:231-6.

12. Grommes C, DeAngelis LM. Primary CNS Iymphoma. J Clin Oncol 2017;35:2410-8.

13. Park JS, Lim DH, Ahn YC, et al. Whole brain radiation dose reduc- tion for primary central nervous system lymphoma patients who achieved partial response after high-dose methotrexate based chemotherapy. Jpn J Clin Oncol 2017;47:995-1001.

14. Sheu T, Milgrom SA, Andraos TY, et al. Response-adapted radiation therapy for newly diagnosed primary diffuse large B-cell lymphoma of the CNS treated with methotrexate-based systemic therapy. Adv Radiat Oncol 2018;3:639-46.

15. Kasenda B, Loeffler J, Illerhaus G, Ferreri AJ, Rubenstein J, Batchelor $\Pi$. The role of whole brain radiation in primary CNS lymphoma. Blood 2016;128:32-6.

16. Omuro AM, Ben-Porat LS, Panageas KS, et al. Delayed neurotoxicity in primary central nervous system lymphoma. Arch Neurol 2005;62:1595-600.

17. Tofilon PJ, Fike JR. The radioresponse of the central nervous system: a dynamic process. Radiat Res 2000;153:357-70.

18. Gondi V, Pugh SL, Tome WA, et al. Preservation of memory with conformal avoidance of the hippocampal neural stem-cell compartment during whole-brain radiotherapy for brain metastases (RTOG 0933): a phase II multi-institutional trial. J Clin Oncol 2014;32:3810-6.

19. Kazda $T$, Jancalek $R$, Pospisil $P$, et al. Why and how to spare the hippocampus during brain radiotherapy: the developing role of hippocampal avoidance in cranial radiotherapy. Radiat Oncol 2014;9:139.

20. Thiel $E_{1}$ Korfel A, Martus $P$, et al. High-dose methotrexate with or without whole brain radiotherapy for primary CNS Iymphoma (G-PCNSL-SG-1): a phase 3, randomised, non-inferiority trial. Lancet Oncol 2010;11:1036-47.

21. Zimmermann M, Oehler C, Mey U, Ghadjar P, Zwahlen DR. Radiotherapy for non-Hodgkin's lymphoma: still standard practice and not an outdated treatment option. Radiat Oncol 2016;11:110.

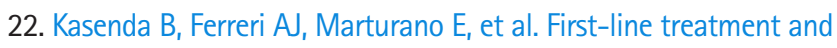
outcome of elderly patients with primary central nervous system lymphoma (PCNSL): a systematic review and individual patient data meta-analysis. Ann Oncol 2015;26:1305-13.

23. Shibamoto $Y$, Hayabuchi N, Hiratsuka J, et al. Is whole-brain irradiation necessary for primary central nervous system lymphoma? Patterns of recurrence after partial-brain irradiation. Cancer 2003;97:128-33.

24. Omuro A, Taillandier $L$, Chinot 0 , et al. Primary CNS Iymphoma in patients younger than 60: can whole-brain radiotherapy be deferred? J Neurooncol 2011;104:323-30. 